\section{FRI0060 MONOCYTE-DERIVED MICROPARTICLES IN CIRCULATION OF PATIENTS WITH RHEUMATOID ARTHRITIS: A NOVEL BIOMARKER FOR THE DISEASE ACTIVITY}

M. Koseki, H. Yasuoka, K. Sakata, K. Yoshimoto, K. Yamaoka, T. Takeuchi. Division of Rheumatology, Department of Internal Medicine, Keio University School of Medicine, Tokyo, Japan

Background: Rheumatoid arthritis (RA) is a systemic connective tissue disease and characterized by inflammation of synovium of multiple joints, however, its pathogenesis is still unclear (1). Physiologically, microparticle (MP) is categorized as a membrane microvesicle which contains various humoral factors such as cytokines or growth factors and 0.1-1 $\mu \mathrm{m}$ in size (2). And MPs can distribute systemically through circulation. MP is released from almost all cell types and induced by stimulation, stress or apoptosis (3). Thus, we hypothesized that MPs derived from various cells can distribute to the lesion of RA and be associated with systemic disease process of RA.

Objectives: To clarify the source of MPs in circulation of RA and elucidate their role in the pathogenesis and the possibility for application as a novel disease marker.

Methods: Twenty patients with RA and 13 healthy controls were involved. Using gradient centrifugation, platelet-rich plasma was isolated from whole blood and applied to flow cytometry. MP is defined as a vesicle less than $1 \mathrm{~mm}$ in diameter and Megamix ${ }^{\circledR}$ was used to set a gating condition to detect microparticles (4). Sources of MP (MP subsets) were identified by the expression of cell-specific markers using flow cytometry with fluorescence-conjugated antibodies as follows; platelets MP: CD $41^{+} \mathrm{CD} 31^{+} \mathrm{MP}$, endothelial cell MP: CD $41^{-} \mathrm{CD} 31^{+} \mathrm{MP}$, immune

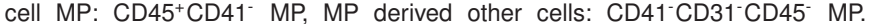
Correlation between clinical information and proportion of MP subsets was also analyzed. Comparisons between two groups were examined by Mann-Whitney $U$-test with Bonferroni correction. Significant difference was defined as corrected $p$ value $(P C)<0.05$

Results: Mean age of 20 patients with RA was $56 \pm 14$ years, $95 \%$ was female, disease duration was $8.5 \pm 8.0$ years, and DAS28-ESR was $2.0 \pm 1.1$. Methotrexate was administered to $75 \%$ and average dose was $8.0 \pm 2.4 \mathrm{mg} / \mathrm{week}$. Plateletderived MP covered the largest proportion in all groups. Interestingly, proportion of immune-cell-derived MP was higher $(P c<0.006)$ in patients with RA compared to controls. Proportion of immune-cell-derived MP was positively correlated with inflammatory markers such as CRP and composite disease markers such as DAS28-ESR. Furthermore, when stratified by monocyte fraction and nonmonocyte fraction based on CD14 expression, proportion of monocyte-derived MP showed higher $(P c<0.01)$ in patients with RA compared to controls, but nonmonocytes MP did not, and that of monocyte-derived MPs was also correlated with disease markers for activity of RA.

Conclusions: Proportion of circulating immune-cell-derived MPs, especially monocyte-derived MPs, increased in RA and correlate with disease activity. These results suggest that monocyte-derived MPs can be measured as a novel biomarker and involved in the disease process of RA.

References:

[1] Smolen JS et al. Lancet 388: 2023-2038, 2016

[2] van der Pol E et al. 64: 676-705, 2012.

[3] Buzas El et al. Nat Rev Rheumatol 10: 356-364, 2014

[4] Chandler WL et al. J Thromb Haemost 9:1216-1224, 2011.

Disclosure of Interest: None declared

DOI: 10.1136/annrheumdis-2017-eular.4599

\section{FRI0061 IDENTIFICATION OF CITRULLINATION SITES SPECIFIC FOR PEPTIDYLARGININE DEIMINASE 2 (PAD2) AND PAD4 IN FIBRINOGEN FROM SYNOVIAL FLUID OF PATIENTS WITH RHEUMATOID ARTHRITIS}

M. Sharma $^{1}$, D. Damgaard ${ }^{2}$, L. Senolt ${ }^{3}$, B. Svensson ${ }^{1}$, A.C.B. Jensen ${ }^{4}$, C.H. Nielsen ${ }^{2}$, P. Hägglund ${ }^{1}$. ${ }^{1}$ Department of Biotechnology and Biomedicine, Technical University of Denmark; ${ }^{2}$ Center for Rheumatology and Spine Diseases, Copenhagen University Hospital, Rigshospitalet, Institute for Inflammation Research, Copenhagen, Denmark: ${ }^{3}$ Department of Rheumatology and Faculty of Medicine, Charles University, Institute of Rheumatology and Department of Rheumatology, Prague, Czech Republic; ${ }^{4}$ Rheumatology, Biomarkers and Research, Nordic Biosciences, Copenhagen, Denmark

Background: Protein citrullination, i.e. conversion of arginine residues into citrulline residues, is a post-translational modification catalyzed by PAD, and is an important pathophysiological determinant in conditions such as Rheumatoid arthritis (RA). Identification of citrullination sites on putative autoantigens is likely to enhance our understanding of PAD's substrate specificity.

Objectives: Citrullinated fibrinogen is an autoantigen linked to the pathophysiology of RA.We have applied a novel MS-based proteomics approach to estimate the degree of citrullination in synovial fluid (SF) from RA patients, and compare to fibrinogen citrullinated in vitro by PAD2/4, the most important PAD isoforms involved in RA. The estimated degree of citrullination induced by the two isoforms is also compared to evaluate their relative impact.

Methods: Fibrinogen was citrullinated in vitro by PAD2/4 and citrullination sites were identified by LC-MS/MS on a Q-exactive orbitrap following proteolytic digestion with Lys-C. These in vitro citrullination profiles were compared to those observed in SF fibrinogen of four RA patients with varying DAS28 scores, CRP levels and leukocyte counts. DAS28 scores $>5.1$ and $\leq 2.4$ correspond to moderate to severe and low activity of disease respectively. Patients with high inflammatory activity gave high CRP level and leukocyte count values.

Results: A total of 52 citrullination sites were identified. Overall, PAD2 generated higher number of identified sites and higher degree of citrulline occupancy at given sites than PAD4. In fibrinogen from SF, 38 citrullination sites were identified, of which 23 have not been previously reported. Several of these sites were identified in more than one patient, and were regarded as hotspots. Fibrinogen from patients with high DAS28 levels contained markedly more citrullination sites and higher citrulline occupancy.

Conclusions: Study suggests that PAD2 citrullinates fibrinogen more efficiently than PAD4 and citrullination of certain sites in fibrinogen from SF reflects disease activity. Identification of such sites may have diagnostic or prognostic value in RA and other inflammatory disorders.

References:

[1] D Makrygiannakis, E af Klint, I E Lundberg, R Lofberg, A-K Ulfgren, L Klareskog, A.I.C. Citrullination is an inflammation-dependent process. Ann Rheum. Dis. 2006, 65 (9), 1219-1222.

[2] Foulquier,C.; Sebbag, M.; Clavel, C.; Chapuy-Regaud, S.; Al Badine, R.; Mechin, M. C.; Vincent, C.; Nachat, R.; Yamada, M.; Takahara, H. Peptidyl arginine deiminase type 2 (PAD-2) and PAD-4 but not PAD-1, PAD-3, and PAD-6 are expressed in rheumatoid arthritis synovium in close association with tissue inflammation. Arthritis Rheum. 2007, 56 (11), 3541-3553.

[3] Gestel, A.; Haagsma, C.; Riel, P. Validation of rheumatoid arthritis improvement criteria that include simplified joint counts. 1998, 41 (10), 1845-1850.

Acknowledgements: The Danish Council for Independent Research Natural Sciences (grant number 11-106246) and the Velux foundation. The Danish Research Foundation, Nordic Bioscience A/S and DTU are acknowledged for a joint PhD scholarship. LS was supported by the Ministry of Health of the Czech Republic (grant \#00023728).

Disclosure of Interest: None declared

DOI: 10.1136/annrheumdis-2017-eular.5950

\section{FRI0062 SYNOVIAL FLUID TREG CELLS SECRETE IL-17 AND AT THE SAME TIME ARE POTENT SUPPRESSORS OF TRESP CELL PROLIFERATION, TNF ALPHA AND IFN GAMMA PRODUCTION}

M. Benito-Miguel ${ }^{1,2}$, A. Villalba ${ }^{1}$, M.-G. Bonilla-Hernán $^{1}$, D. Peiteado ${ }^{1}$

A. Balsa ${ }^{1}$, P. Aguado ${ }^{1}$, P. Sánchez-Mateos ${ }^{3}$, A. Puig-Kröger ${ }^{3}$, E. Martin-Mola ${ }^{1}$ M.E. Miranda Carus ${ }^{1}$. ${ }^{1}$ Rheumatology, HOSPITAL LA PAZ - IdiPAZ;

${ }^{2}$ Biochemistry, Centro de Ciencias de la Salud San Rafael. Universidad Antonio de Nebrija; ${ }^{3}$ Immunology, Hospital Gregorio Marañon, Madrid, Spain

Background: IL-17-expressing FoxP3 regulatory $\mathrm{T}$ cells have been described, and their suppressive capacity has been questioned. An inflammatory environment seems to favor IL-17 secretion by regulatory CD4+CD25+FoxP3+ T cells.

Objectives: To assess the suppressive function and IL-17 producing capacity of CD4+CD25+CD127-FoxP3+ T cells in the synovial fluid of RA patients (RASFd).

Methods: Synovial fluid was drawn from 35 patients with established RA who were receiving methotrexate and low-dose oral prednisone. The frequency of CD4+CD25+CD127-FoxP3+ T cells was assessed by flow cytometry. Total CD4+ $\mathrm{T}$ cells, CD4+CD25+CD127- $\mathrm{T}$ reg cells and CD4+CD25- Tresp cells were isolated by Ficoll-Hypaque gradient, followed by sorting. After isolation, cells were stimulated for 5 hours with PMA+ionomycin or cultured for 5 days in flat-bottom 96-well plates coated with an anti-CD3 monoclonal antibody. Treg cell function was assessed using two different approaches: A. The regulatory function of natural proportions of Tregs was inferred by comparing the proliferative and cytokine responses of total CD4+ $T$ cells (TCD4T) versus CD25+ depleted CD4+ T cells (CD4+CD25-T cells or Tresp cells); B. The per cell suppressor potency of Tregs was assessed in cocultures of isolated Tregs with Tresp, established at different Treg/Tresp ratios. Proliferation was determined by 3 Hthymidine incorporation and CFSE dilution; cytokine secretion was measured by ELISA of culture supernatants.

Results: A high proportion of CD4+CD25+CD127- T cells was present in RASFd (mean \pm SD, $21.1 \% \pm 6.2$ ), which is significantly higher than reported frequencies of this cell population in the peripheral blood of both RA and healthy subjects. These RASFd CD4+CD25+CD127- T cells expressed FoxP3 but did not express CD69. The proliferation rate, TNF $\alpha$ and IFN $\gamma$ secretion were significantly higher for isolated Tresp as compared with TCD4T cells, indicating that natural proportions of Treg cells present in the synovial fluid of RA are funcionally suppressive. Surprisingly, TCD4T cells secreted higher amounts of IL-17 as compared with Tresp cells, although the difference did not reach statistical significance. On a per cell basis, RAPB Tregs were potent suppressors of Tresp proliferation, TNF $\alpha$ and IFN $\gamma$ but not of IL-17 secretion; in fact IL-17 secretion did not decrease but was enhanced in the presence of increasing proportions of Treg cells. Isolated Treg cells did not proliferate or produce cytokines when cultured alone in anti-CD3 coated plates. However, in the presence of plate-bound anti-CD3 plus anti-CD28 and recombinant human IL-2, isolated Treg cells secreted significant amounts of $\mathrm{IL}-17$ whereas no TNF $\alpha$ or IFN $\gamma$ could be detected in supernatants.

Conclusions: CD4+CD27+CD127- FoxP3+ Treg cells present in the synovial fluid of RA patients are potent suppressors of Tresp proliferation, TNF $\alpha$ and IFN $\gamma$ secretion, and at the same time produce significant amounts of IL-17. 
References:

[1] Voo KS, et al. Proc Natl Acad Sci U S A. 2009;106:4793.

[2] Beriou G et al. Blood. 2009;113:4240.

[3] Wang T, et al. Ann Rheum Dis. 2015;74:1293.

[4] Komatsu N, et al. Nat Med. 2014;20:62.

[5] Yang BH, et al. Mucosal Immunol. 2016;9:444.

Disclosure of Interest: None declared

DOI: 10.1136/annrheumdis-2017-eular.4088

\section{FRI0063 RESVERATROL ATTENUATES SYNOVIAL HYPERPLASIA IN AN ACUTE ANTIGEN-INDUCED ARTHRITIS MODEL BY AUGMENTING AUTOPHAGY AND DECREASING ANGIOGENESIS}

M. Almonte-Becerril ${ }^{1}$, J.A. Fernandez-Rodriguez ${ }^{1}$, J.A. Fernandez-Rodriguez ${ }^{1}$, O. Ramil-Gomez ${ }^{1}$, R.R. Riveiro-Naveira ${ }^{1}$, L. Hermida-Carballo ${ }^{1}$, A. Concha ${ }^{2}$, A. Vela-Anero ${ }^{3}$, S. Viñas ${ }^{1}$, F.J. Blanco ${ }^{4}$, M.J. Lopez-Armada ${ }^{1} .{ }^{1}$ Aging and Inflammatory Research Lab; ${ }^{2}$ Servicio de Patología; ${ }^{3}$ Terapia Celular y Medicina Regenerativa; ${ }^{4}$ Laboratorio de Investigacion Osteoarticular y Envejecimiento, SERGAS, A Coruña, Spain

Background: Previously, we have demonstrated that dietary supplementation with resveratrol lowers synovial hyperplasia, inflammatory markers and oxidative damage in an acute antigen-induced arthritis (AIA) model.

Objectives: In this work, we investigated whether resveratrol can also regulate this abnormal proliferation of synovial tissue in an acute AIA model by inducing cell death pathways and by modifying the angiogenesis in the synovial membrane. Methods: Animals were randomly divided into 3 groups: control, AIA, and resveratrol-treated AIA group. Resveratrol $(12,5 \mathrm{mg} / \mathrm{kg} /$ day $)$ was given orally 8 weeks before AIA induction until sacrifice day ( $48 \mathrm{~h}$ after intra-articular injection). Control and AIA animals were administered $100 \mu \mathrm{l}$ of water. Resveratrol effects on autophagy and apoptosis were evaluated by LC3 and active caspase-3 expression (confocal and immunohistochemistry, respectively). Angiopoietin 1 (Ang-1), vascular endothelial growth factor (VEGF), and the nuclear factor NFkappa-B p65 subunit (p65) were also determined by immunohistochemistry and cartilage degradation with Safranin-O.

Results: Resveratrol significantly reduced the histological score of synovial tissue. Results showed a significant higher expression of LC3 signals in the AIA synovial membranes, compared with control samples, in which the presence of vesicles was easily observed. Interestingly, the synovial tissues from the resveratrol group showed a significantly $(p \leq 0.001)$ higher signal for LC3, compared with the AIA samples. Active caspase-3 expression was up-regulated at the same level in the synovial membranes of AIA group than in resveratrol-treated AIA group; however, in resveratrol-treated AIA group active caspase- 3 signal was mainly located in the inflammatory cells. Resveratrol consumption significantly attenuated Ang-1 signal, whereas expression of VEGF showed a non significant reduction. Resveratrol administration also mitigated, even not significantly, p65 expression that was significantly higher in the AIA animals than those from the control animals. In addition, resveratrol decreased articular cartilage degradation.

Conclusions: These data suggest that resveratrol is able to modulate synovial hyperplasia by increasing autophagic cell death and limiting angiogenic response in an acute AIA model, which could also modulate the inflammatory and destructive processes for rheumatoid arthritis.

Acknowledgements: Instituto de Salud Carlos III-Ministerio de Economía y Competitividad N Expediente PI12/02771. Unión Europea-Fondo Europeo de Desarrollo Regional (FEDER) "Una manera de hacer Europa"

Disclosure of Interest: None declared

DOI: 10.1136/annrheumdis-2017-eular.6270

\section{FRI0064 CHEMOKINE RECEPTOR 6 MODULATES ARTHRITIS IN A T CELL DEPENDENT MANNER}

M. Bonelli, A. Puchner, L. Goeschl, S. Hayer, B. Niederreiter, J.S. Smolen, C. Scheinecker, S. Blueml. Devision of Rheumatology, Medical University of Vienna, Vienna, Austria

Background: Rheumatoid arthritis (RA) is an inflammatory autoimmune disease, characterized by synovial infiltration of various cells. Chemokines are involved in the recruitment of different cell types into the synovial membrane. Accumulation of CCR6 expressing mononuclear cells can be found in joints of RA patients. CCR6 expression has also been reported on $\mathrm{CD} 4^{+} \mathrm{T}$ cells, in particular regulatory as well as Th17 cells. In addition, a subset of regulatory $\mathrm{T}$ cells, namely CD25-Foxp3 ${ }^{+}$T cells, can upregulate CCR6 and RANKL and thereby can promote osteoclastogenesis.

Objectives: In this study, we investigated the role of CCR6 in the pathogenesis of arthritis using different arthritis models.

Methods: Clinical as well as histological signs of arthritis were investigated in the collagen-induced arthritis (CIA), K/BxN serum transfer arthritis and in the human tumor necrosis factor (hTNFtg) arthritis model, comparing wt and CCR6-/- mice. We analyzed the phenotype of lymph node cells by flow cytometry and cytokine concentrations in serum. Anti-collagen antibodies and cytokines were measured by enzyme-linked immunosorbent assay.

Results: The K/BxN serum transfer arthritis and hTNFtg arthritis model are known to be T cell independent. Since CCR6 is an important component of the innate immune system we compared the development of arthritis in both models. We did not detect any significant differences in clinical signs of inflammation or histological severity of arthritis between wt and CCR6-/- mice. In addition, bone volume was similar between $w t$ and CCR6-/- mice. To investigate the role of CCR6 as part of the adaptive immune system in the development of arthritis we induced CIA in wt and CCR6-/- mice, which is known to be T cell dependent. CCR6-/- mice were almost completely protected from CIA. Indeed, analyses of T cell subsets by flow cytometry revealed a significant reduction of $\mathrm{CD}^{-} 5^{-} \mathrm{Foxp}^{+} \mathrm{T}$ cells.

Conclusions: CCR6 is necessary for the generation of pathogenic $\mathrm{CD}^{2} 5^{-} \mathrm{Foxp}^{+}$ T cells in CIA, suggesting an important function of CCR6 on T cells in the development of autoimmune arthritis.

Disclosure of Interest: None declared

DOI: 10.1136/annrheumdis-2017-eular.5107

\section{FRI0065 HELMINTHES BASED NOVEL COMPOUND, TUFTSIN-PHOSPHORYLCHOLINE (TPC) AMELIORATES ESTABLISHED MURINE ARTHRITIS}

T. Bashi ${ }^{1}$, J. Lachnish ${ }^{1}$, M. Fridkin ${ }^{2}$, Y. Shoenfeld ${ }^{3}$, M. Blank ${ }^{1} .{ }^{1}$ Zabludowicz Center for Autoimmune Diseases, Sheba Medical Center, Ramat Gan; ${ }^{2}$ Organic Chemistry, Weizmann, Rehovot; ${ }^{3}$ Zabludowicz Center for Autoimmune Diseases, Sheba.health.gov.il, Ramat Gan, Israel

Background: In areas where helminthes infections are common, autoimmune diseases are rare. Treatment with helminthes or their ova, improved clinical findings of several autoimmune diseases. Based on the helminthes biological activity, a novel chimeric be-specific molecule was synthetized of tuftsin-phosphoryl-choline (PC)-TPC.

Objectives: To study TPC treatment in established collagen induced arthritis (CIA) mice, and the mechanism of activity.

Methods: Arthritis was induced in DBA male mice by immunization with collagen emulsified in TB-mycobacteria at the tail base. Boost was given 3 weeks later. Treatment with TPC started when the clinical score was 2. Cytokines were measure in culture-fluid of splenocytes in-vitro. T regulatory cells and B regulatory cells were measured by FACS. TPC effect on TLR4 expression was studied using HEKTM-mTLR4 cells system and its inhibitor. M1 shift to M2 was performed in RAW macrophages differentiated to M1 by PMA followed by LPS. TPC was added and IL-6, TNFalpha, IL-10 were tested by ELISA.

Results: Starting TPC treatment of CIA mice after disease establishment, had a significant lower arthritis score in comparison with control vehicle subjected mice (i.e. TPC- $6.8 \pm 0.8$ vs vehicle- $13.8 \pm 0.45 ; p<0.0001$ ). Joints staining revealed normal joint structure in TPC treated mice, whereas, control mice treated with PBS, PC or tuftsin had severe inflamed joints. Likewise, TPC enhanced antiinflammatory response by enhanced IL-10 secretion, reduced pro-inflammatory cytokines secretion (IL-1 $1, \mathrm{IL}-17, \mathrm{IL}-6$, and TNF- $\alpha)(\mathrm{p}<0.001)$. Furthermore, TPC induced expansion of splenic CD4+CD25+FOXP3+ T regulatory cells (Tregs) and IL-10+CD5+CD1d+B regulatory cells (Bregs). The mechanism underling the TPC related immunomodulatory activity was attributed to its bi-specific activity: a) Shift of Raw cells macrophages from pro-inflammatory macrophages M1 to anti-inflammatory M2 secreting anti- IL-10 $(p<0.001)$, through the tuftsin part of TPC. b) TPC inhibited significantly TLR4 expression by HEKTM-mTLR4 cells $(p<0.02)$ via the phosphorylcholine end. Our data indicated that TPC significantly ameliorated established CIA by anti-inflammatory immunomodulatory activity.

Conclusions: Our data may lead to a novel bi-specific self small molecule for therapy of patients with advanced RA.

Disclosure of Interest: None declared

DOI: 10.1136/annrheumdis-2017-eular.3065

\section{FRI0066 GASTRIN-RELEASING PEPTIDE AND ITS RECEPTOR INCREASE ARTHRITIS FIBROBLAST-LIKE SYNOVIOCYTE INVASIVENESS OVER THE PI3K/AKT PATHWAY}

M. Farinon ${ }^{1,2}$, V.S. Clarimundo ${ }^{1}$, R.T. Pedó ${ }^{1,2}$, V.O.N. Teixeira ${ }^{1}$, C. Nör ${ }^{3}$, P.S. Gulko ${ }^{4}$, R.M. Xavier ${ }^{1,2}$, P.G. Oliveira ${ }^{1,2}{ }^{1}$ Hospital de Clínicas de Porto Alegre; ${ }^{2}$ Universidade Federal do Rio Grande do Sul, Porto Alegre, Rio Grande do Sul, Brazil; ${ }^{3}$ Peter Gilgan Centre for Research and Learning, Toronto, Ontario, Canada; ${ }^{4}$ Icahn School of Medicine at Mount Sinai, New York, United States

Background: Rheumatoid arthritis (RA) is an autoimmune disease where the chronic inflammation and subsequent cartilage and bone erosion lead to joint destruction. The fibroblast-like synoviocytes (FLS) have a central role in disease pathogenesis and in vitro FLS invasiveness correlates with articular damage in RA patients. Gastrin-releasing peptide (GRP) plays an important role in the immune and inflammatory response. GRP is found in synovial fluid of RA patients and its receptor (GRPR) is found in synovial membrane of murine arthritis. RC-3095 in an antagonist of GRPR.

Objectives: To evaluate the role of GRP and GRPR on invasive behavior of mice FLS and to evaluate the GRP-induced signaling on PI3K/AKT pathway.

Methods: FLS were isolated from joints of DBA/1J mice with collagen-induced arthritis. Expression of GRPR in FLS was investigated by immunocytochemistry and western blot (WB). Proliferation of FLS treated with GRP $(0.1 \mu \mathrm{M}-10 \mu \mathrm{M})$ 